unchanged Leuchs anhydride was recovered. In its solutions, the anhydride is much less stable and polymerization takes place readily, especially on heating.

The purified polymers obtained give a biuret reaction on prolonged standing ; they are practically insoluble in the usual solvents, including dilute acids and alkali, but partly soluble in anhydric formic acid, glacial acetic acid, dimethyl formamide and dichloroacetic acid.

In the course of the present experiments the diketopiperazine derivative (VI) was also obtained from the ethyl ester (V).

The experiments described here are part of an investigation on the polymerization of amino-acids and their derivatives, containing unsaturated bonds, the results of which will be published at a later date.

Department of Organic Chemistry, Max Frankex Albert Zilkha

The Hebrew University, Jerusalem. Jan. 25.

1 Stoll, A., and Seebeck, E., Helv. Chim. Acta, 31, 189 (1948)

\section{Proof of the Heisenberg Relations}

SiNCE Heisenberg's uncertainty relations play an essential part in quantum mechanics, especially in the discussion of the dual wave-particle aspect of matter, the derivation of these relations is of importance. We became interested in this problem in connexion with a lecture course on quantum mechanics given by one of us. In this course we wished to give a derivation of the Heisenberg relations which was rigorous without, however, involving commutator relations.

In the literature we found that, of the many textbooks in the field, some give only an outline of a proof, some give a qualitative proof based on the analogy between wave mechanics and optics, using the properties of wave trains, some use commutator relations, while Moyal ${ }^{1}$ and Weyl use the Schwartz inequality. The well-known proof due to Heisenberg ${ }^{2}$, which is also given by Pauli and Rojanski, is the kind of proof we had in mind. Unfortunately, this proof is not completely rigorous, and it is the object of this communication to modify Heisenberg's proof slightly to make it so.

Restricting ourselves to the discussion of the onedimensional case, let $\psi(x)$ be the normalized wave function of the electron with its Fourier expansion,

$$
\psi(x)=\int A(\sigma) \exp (2 \pi i \sigma x) d \sigma
$$

where $x$ is the position of the electron, and $\sigma$ the wave number. We have from equation (1),

$$
A(\sigma)=\int \psi \exp (-2 \pi i \sigma x) d x
$$

We introduce now the mean position, $x_{0}$, the mean wave number, $\sigma_{0}$, the uncertainty in position, $\Delta x$, and the uncertainty in wave number, $\Delta \sigma$, by the equations,

$x_{0}=\left.\int x_{1}^{\prime} \psi\right|^{2} d x, \quad \sigma_{0}=\int \sigma|A|^{2} d \sigma$,

$\Delta x^{2}=\int\left(x-x_{0}\right)^{2}|\psi|^{2} d x, \quad \Delta \sigma^{2}=\int\left(\sigma-\sigma_{0}\right)^{2}|A|^{2} d \sigma$

We choose the origin of the $x$ and $\sigma$ scales in such a way that $x_{0}=\sigma_{0}=0$, so that the last two equations (3) become

$$
\begin{gathered}
\Delta x^{2}=\int x^{2}|\psi|^{2} d x, \\
\Delta \sigma^{2}=\int \sigma^{2}|A|^{2} d \sigma=(4 \pi)^{-2} \int\left(\frac{\partial \psi}{\partial x}\right)^{2} d x
\end{gathered}
$$

the last equation following in the usual way.

It is clear that the following inequality will always hold, irrespective of the choice of constant $a$ :

$$
|a x \psi+(\partial \psi / \partial x)|^{2} \geqslant 0
$$

From expression (5) it follows by the usual analysis that

$$
a^{2} \Delta x^{2}+4 \pi^{2} \Delta \sigma^{2} \geqslant a
$$

At this point, Heisenberg puts $a=\left(2 \Delta x^{2}\right)^{-1}$ and thus obtains the Heisenberg relations. There is, however, no obvious reason for this choice of $a$, and by choosing a different value one could derive relations between $\Delta x$ and $\Delta \sigma$ which would be completely different from the Heisenberg relations.

The proof of the Heisenberg relations, however, can be completed in the following way. From inequality (6) we see that the smaller $\Delta x^{2}$ is, the larger, in general, will be $\Delta \sigma^{2}$ and that the smallest combination occurs when the equality sign holds, that is, when $\psi$ satisfies the equation,

$$
a x \psi+(\partial \psi \partial / x)=0
$$

The wave function satisfying equation (7) is of the Gaussian form and $a$ is now related to $\Delta x^{2}$ by the equation $a=\left(2 \Delta x^{2}\right)^{-1}$; but this is not necessarily true for a different form of $\psi$. Substituting this value of $a$ into equation (6), we get,

$$
\Delta \sigma \cdot \Delta x=1 / 4 \pi
$$

and since the situation is less favourable when $\psi$ does not satisfy equation (7), we get in general $\Delta \sigma . \Delta x \geqslant$ $1 / 4 \pi$ as before.

D. TER HAAR

W. M. NICOL

Department of Natural Philosophy,

University, St. Andrews.

Jan. 19.

${ }^{1}$ Moyal, J. E., Proc. Camb. Phil. Soc., 45, 119 (1949).

"Heisenberg. W. "Die Physikalischen Prinzipien der Quantentheorie" (Htrzel Verlag, Leipzig, 1930)

\section{Germination of Rice Embryo under Water and its Relation of Growth to Endosperm Fractions}

IT is a well-known fact that rice seeds are capable of germination under water and the coleoptile of the rice embryo grows more rapidly and more extensively under water than in air. Yamada ${ }^{1}$ suggested that the superior growth of the rice coleoptile under water is due to the decreased capacity of the coleoptile tissue for destroying auxin under water as compared to the extensive capacity of the tissue for destroying auxin in air. Sircar and Das ${ }^{2}$ have shown that the auxin of the rice endosperm gradually decreases with the growth of the coleoptile and root; such gradual consumption of auxin is stopped abruptly when the embryo is detached from the endosperm.

It has been observed that some factor present in the endosperm has a distinct inhibitory effect on the growth of the rice embryo kept under water or grown in air. Experiments were carried out with husked rice grain (variety Bhasamanik) from which different fractions of endosperm were removed, kept under water and on agar slopes in air in darkness at $25^{\circ} \mathrm{C}$. 